\title{
Comparative Study of Load Balancing Techniques in Mobile WiMAX IEEE 802.16e
}

\author{
Ahmed S. Abdel-Rahman, Noura Semary, Hatem S. Abdelkader \\ Department of Information Technology, Department of Information Systems \\ Faculty of Computers and Information \\ Menoufia University, Egypt \\ ahmed.samy@ci.menofia.edu.eg,noura.samri@ci.menofia.edu.eg,Hatem.abdelkader@ci.menofia.edu.eg
}

\begin{abstract}
Mobile Worldwide Interoperability for Microwave Access (Mobile WiMAX) is a wireless Metropolitan Area Network technology based on IEEE 802.16e standard. Mobile WiMAX has been designed with the purpose of enabling mobile Internet from the physical layer to the network layer. In this paper, a comparative study between load balancing techniques in mobile WiMAX is presented. These techniques are Spare Capacity Procedure (SCP) and WiMAX QOS Aware Load Balancing Protocol (WQLP). These load balancing techniques used when network congestion occurred in Mobile WiMAX networks. These two techniques represent the major trends of load balancing in Mobile WiMAX IEEE 802.16e technology. These techniques use directed handovers for Load Balancing (LB) among cells; these handovers were initiated by the Serving Base Station. Performance of load balancing techniques have been analyzed and evaluated based on an extensive simulation. The simulation shows that the performance of WQLP is better than SCP in load distribution but SCP is faster than WQLP in handover process. The elaborated performance analysis shows a set of advantages and disadvantages of these techniques. This evaluation study represents an important entry point for choosing the best techniques that can distribute load among BSs and guarantee QoS for all MSs using real-time applications.
\end{abstract}

Keywords-Mobile WiMAX; Load Balancing; Forced Handover; Quality of Service.

\section{INTRODUCTION}

Growing demand in the whole world on high speed broadband wireless networks led to motivate the development of new technologies in the field of wireless networks. These technologies support high speed streaming multimedia, customized personalized services, ubiquitous coverage and QoS. WiMAX appears to be one of the most promising technologies in the last few years. It is based on IEEE 802.16e-2005 amendment to the 2004 release standard [ HYPERLINK VI "4699489" 1 ]. The Orthogonal Frequency-Division Multiplexing (OFDM) based IEEE 802.16d 2] technology provides fixed broadband access from anywhere within a metropolitan area network. The new mobile air interfaces specified in the IEEE 802.16e [ HYPERLINK 1 "1603394" 3 ] has successfully addressed the requirements for higher data rates and efficient spectral use. The IEEE 802.16e standard, known as Mobile WiMAX, extends WiMAX to support mobility and wireless multimedia services. Mobile WiMAX allows Mobile Station (MS) to handover from the Serving Base Station (SBS) to a one of its neighbor BSs called the TBS. The IEEE 802.16 standardization group has defined three types of approaches towards handover for the 802.16e technology 4]; the default procedure called Hard Handover (HHO). The other procedure called Soft Handover (SHO). There are two optional types of soft handover called Fast Base Station Switching (FBSS) and Macro Diversity Handover (MDHO) [ HYPERLINK 1 "5455786" 4 ]. In WiMAX, a handover initiation decision is taken by Mobile Station or by SBS. This decision depends on the Received Signal Strengths (RSS) from the current SBS and NBS. The HHO is a Break-Before-Make procedure 5], in which the MS breaks its connection with the SBS then makes a new connection with the TBS. HHO is less complex, high latency and not suitable for real-time applications. In MDHO the MS allowed to keep a valid connection in the same time with more than one BS. MDHO is a complex soft handover technique and needs prolonged amount of signaling between MS and all BSs in the Active Set. In FBSS the MS monitors a set of BSs permanently and maintains the Active Set. The MS may perform several connections with the BSs of the Active Set but MS allowed to communicating with only one BS called the anchor BS.

Wasting channel resources, handover latency time and data loss are existing drawbacks in the WiMAX handover techniques [ HYPERLINK V1 "tag2011wimax" 6 ]-7]. QoS and resource scheduling become an important issue in Mobile WiMAX. Customer required service can be mapped onto one of the following scheduling service classes [ HYPERLINK V1 "2000" 8 ]. The real-time Polling Service (rtPS) that used with applications such as MPEG video service. The Extended Real Time Polling Service (ertPS) supports real-time uplink service flow transporting periodically variable size data packets such as VoIP traffic with silence suppression. Non-real-time Polling Service (nrtPS), this service class is suitable for non-real time applications such as FTP traffic. Unsolicited Grant Service (UGS) that used with applications such as the Voice over IP (VoIP) calls and Best Effort 
(BE) can serve data flows without QoS constraints. MAC layer is responsible for packet scheduling, handover, admission control and load balancing mechanism. Many QoS and Load Balancing techniques left unspecified in Mobile WiMAX amendments. Researchers start solving these drawbacks by offering some mechanisms to solve these drawbacks that best suited to these particular requirements. Optimal allocation and utilization of radio resources between MS and BS is a major challenge 9]. When congestion occurred in the network, MS service degraded. So, load balancing techniques are introduced to achieve this goal using BS initiated direct Handover (HO) to force MS to migrate from the air-interface provided by SBS to the air-interface of another Target BS.

The rest of this paper is organized as follows: Section II, load balancing techniques used in Mobile WiMAX networks have been discussed. Result has been discussed and analyzed using OPNET Modeler 17.1 in Section III. Conclusion has been drawn in Section IV.

\section{LOAD BALANCING TECHNIQUES USED IN MOBILE WiMAX}

In Mobile WiMAX networks, Network congestion occurred when load on network increased in rush hours at popular places. For example, large numbers of Mobile Station (MS) nodes come to a specific work area every morning. If all MS nodes in this area used network resources or run real-time applications, Serving Base Station (BS) becomes congested and overloaded. So, BS resources decreased and affected the performance of real-time applications and MSs admitted service flows decreased. There are two trends for load balancing in Mobile WiMAX; the first trend is to distribute load among BSs based on the empty resources such as free slots in the uplink and downlink, the second trend based on a set of QoS parameters for five QoS classes used in Mobile WiMAX. Spare Capacity Procedure technique [ HYPERLINK V "4699489" 1 ] is chosen to represent the first trend and WiMAX QoS Aware Load Balancing technique is chosen 10] to represent the second trend. A few paper discussed a load balancing with handover in IEEE 802.16e base system. In [ HYPERLINK V "4151342" 11 ] an advanced load balancing technique proposed based on network controlled handover. However, the deployment of this algorithm requires extensive amount of signaling and presence of a centralized architecture is preferred. An inter-frequency reuse handover among frequency assignments is suggested to solve unbalanced load distribution in IEEE 802.16e networks. This scheme is not controlled by network [12]. In 13] the proposed algorithm use dual handover mechanism where MS and BS initiated handovers are used adaptively. However, this procedure is not suitable for real time applications. A novel handover algorithm to balance the load of the layers in a multi-reuse scenario in mobile WiMAX systems is proposed in [ HYPERLINK \l "5594568" 14]. In 15] a new distributed uplink packet scheduling algorithm in WiMAX networks is proposed. The uplink available resources estimated for each connection in this algorithm to provide required resources and guarantee QoS requirements of each connection in the same time.

Spare Capacity Procedure (SCP) technique chosen to represent the first trend of load balancing, and WiMAX QoS Aware Load Balancing Protocol (WQLB) chosen to represent the second trend of load balancing.

\section{A. Spare Capacity Procedure (SCP)}

In [ HYPERLINK V "4699489" 1 ], a load balancing scheme proposed for load balancing in Mobile WiMAX. This scheme used Spare Capacity Report (SCR) among BSs; Spare Capacity is described as Uplink and Downlink available radio resources in each BS. SCR can also include the total number of Uplink and Downlink slots in the frame; the actual amount of available resources in the TBS is known by BS that initiate the Load Balancing. SCR broadcasted every Load Balancing Cycle (LBC) among SBS and NBSs. The spare capacity information received in the SCR used as an indicator of the feasible set of TBS. The BS computes $\mathrm{L}$ and $\mathrm{T}$ when receiving SCR from neighbor BS; T is a Threshold calculated from Eq. 1

$$
T=L+\delta L
$$

where $\mathrm{L}$ is an average load level and $\delta$ is hysteresis margin used to avoid "Ping-Pong" effect. Load-states are underloaded, balanced and overloaded. The BS load-state is determined based on conditions in Table I where U represents BS load. SBS applies load balancing if load-state becomes overloaded, the BS will initiate directed handovers for MSs that reside in overlapping areas or sending MOB_SCN_RSP message to MSs that will perform handover.

TABLE I. BS LOAD STATE CONDITIONS

\begin{tabular}{|c|c|}
\hline Load State & Condition \\
\hline Underloaded & $\mathrm{U}<\mathrm{L}$ \\
\hline Balanced & $\mathrm{L}<\mathrm{U}<\mathrm{T}$ \\
\hline Overloaded & $\mathrm{U}>\mathrm{T}$ \\
\hline
\end{tabular}




\section{A. S. Abdel-Rahman, N. Semary, H. S. Abdelkader}

The directed handover procedure will executed as illustrated in Fig. 1. SBS then sends HO_REQ message to TBS, if TBS load-state is underloaded it will accept the directed handover. If TBS load-state is balanced or overloaded, it will reject the directed handover but it will accept rescue handover. Rescue handover in 10] is defined as when the MS receive signal strength less than pre-defined threshold then MS will inform its SBS that their link is going down and it needs to switch to another BS. This technique can prevent "Ping-Pong" effect using hysteresis margin and can balance the load of the system but it doesn't take into account the service level and QoS for MSs that will execute directed HO.

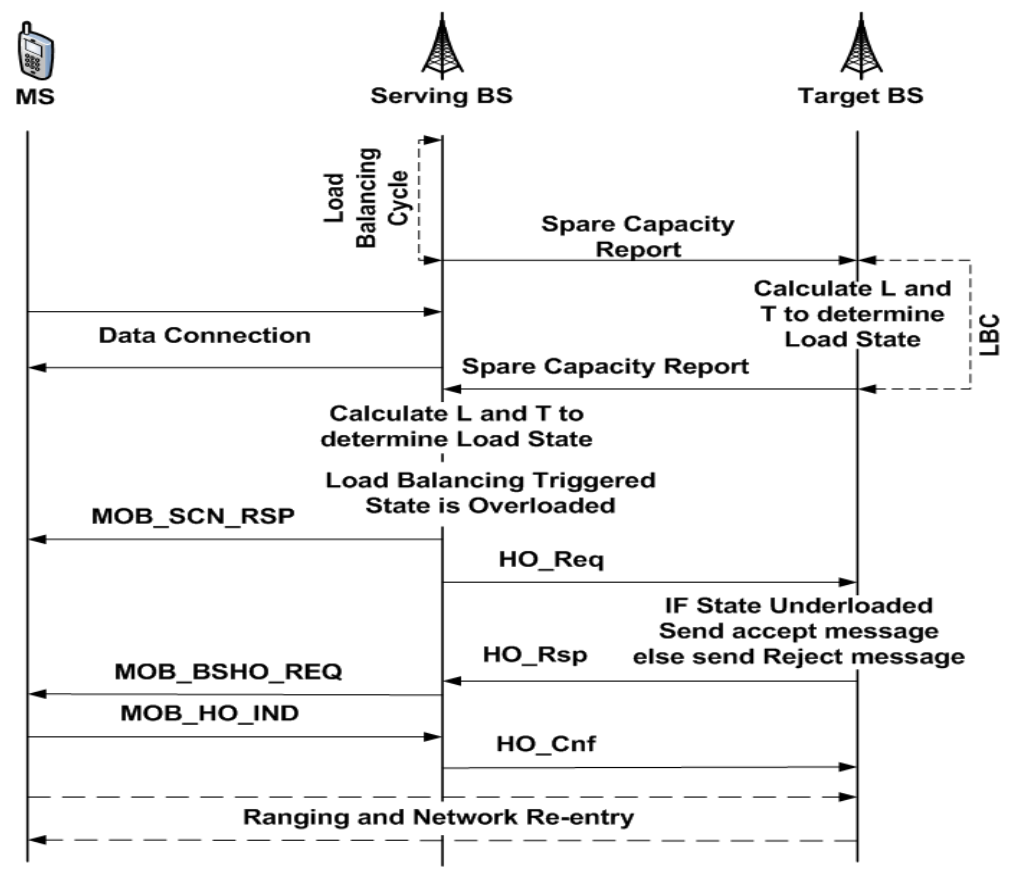

Fig. 1. Mobile WiMAX Load Balancing in SCP Scheme.

\section{B. WiMAX QoS Aware Load Balancing Protocol (WQLB)}

A QoS aware scheme with forced handover proposed for load balancing in Mobile WiMAX in [ HYPERLINK $\mathrm{I}$ "amiri2012wqlb" 10 ]. This scheme focused on load definition, overload detection, and handover operation processes. Load state defined based on the performance parameters of the five service classes namely UGS, rtPS, ertPS, nrtPS and BE in Mobile WiMAX. These parameters are throughput in uplink and downlink, delay and jitter. IEEE 802.16e determines five different scheduling services with its QoS parameters as shown in Table II 16]- [ HYPERLINK Vl "2011wimax" 17 ]. Delay and jitter are the two important criteria in real time application services rtPS, ertPS, and UGS. Delay is specified as how long it takes for a data packet to travel across the network from one MS to destination node or in an opposite direction. Jitter is expressed as a time difference in packet inter-arrival time to their destination. In overload detection the proposed method takes all of performance parameters of QoS classes into account and compares each of them with their relevant thresholds and also their associated hysteresis margin to avoid handover "Ping-Pong" effect.

TABLE II. The Scheduling SERVICE OF Mobile WiMAX.

\begin{tabular}{|c|c|c|c|c|c|}
\hline $\begin{array}{c}\text { QoS Classes and } \\
\text { Parameters }\end{array}$ & UGS & rtPS & ertPS & nrtPS & BE \\
\hline $\begin{array}{c}\text { Minimum Reserved } \\
\text { Traffic Rate }\end{array}$ & & $\sqrt{ }$ & $\sqrt{ }$ & $\sqrt{ }$ & \\
\hline $\begin{array}{c}\text { Maximum Sustained } \\
\text { Traffic Rate }\end{array}$ & $\sqrt{ }$ & $\sqrt{ }$ & $\sqrt{ }$ & $\sqrt{ }$ & $\sqrt{ }$ \\
\hline Maximum Traffic Burst & & $\sqrt{ }$ & $\sqrt{ }$ & $\sqrt{ }$ & \\
\hline Tolerated Jitter & $\sqrt{ }$ & & $\sqrt{ }$ & & \\
\hline Maximum Latency & $\sqrt{ }$ & $\sqrt{ }$ & $\sqrt{ }$ & & \\
\hline
\end{tabular}


In a handover operation some of MS nodes residing in the overlapping area are chosen to be transferred to neighboring BS. Every LBC the SBS compares its Load matrix (LM) with the Threshold matrix (TH) and Hysteresis Margin matrix (HM) where

$$
[T H]=[T H]+([T H] *[H M])
$$

If elements of load matrix of BS (LMBS) have passed the TH matrix of the above formula, BS notifies that it is in an overload state. If the SBS is in overload state, it finds MSs residing in overlapped area to switch its connection to TBS. Since BSs are communicating with each other through ASN gateways, they know their adjacent BSs situations in advance. Chosen MSs in the candidate list then execute Forced Handover to TBS.

\section{EVALUATION OF LOAD BALANCING TECHNIQUES}

In this section, the two load balancing techniques for Mobile WiMAX have been evaluated. These techniques use forced handover to distribute load among neighbor BSs. These two load balancing techniques are evaluated. We use OPNET Modeler 17.1 to simulate these two techniques. OPNET Modeler is extensive and powerful simulation software with wide variety of possibilities to simulate entire heterogeneous networks with various protocols 18]. The OPNET Modeler environment incorporates tools for all phases of a study, including model design, simulation, data collection, and data analysis. The open source network simulator has the advantage that everything is very open and everyone or organization can contribute to it and find bugs in it.

The simulated WiMAX network is composed of three WiMAX BSs forming three cells connected via backbone connection as shown in Fig. 2, Blue cell in top has 5 MS nodes, Red cell in bottom has 3 MS nodes and Green cell in right has 3 MS nodes. All MS nodes are running voice traffic over UGS connections (in the uplink). The physical profile used for MS nodes and BS is Wireless Orthogonal Frequency-Division Multiple Access (OFDMA) 20 MHZ, QPSK 1/2 for Modulation and Encoding. The serve is the destination for all MS nodes traffic, which has VOIP application with Pulse-code modulation (PCM) Quality Speech and Simulation run for 1 hour.

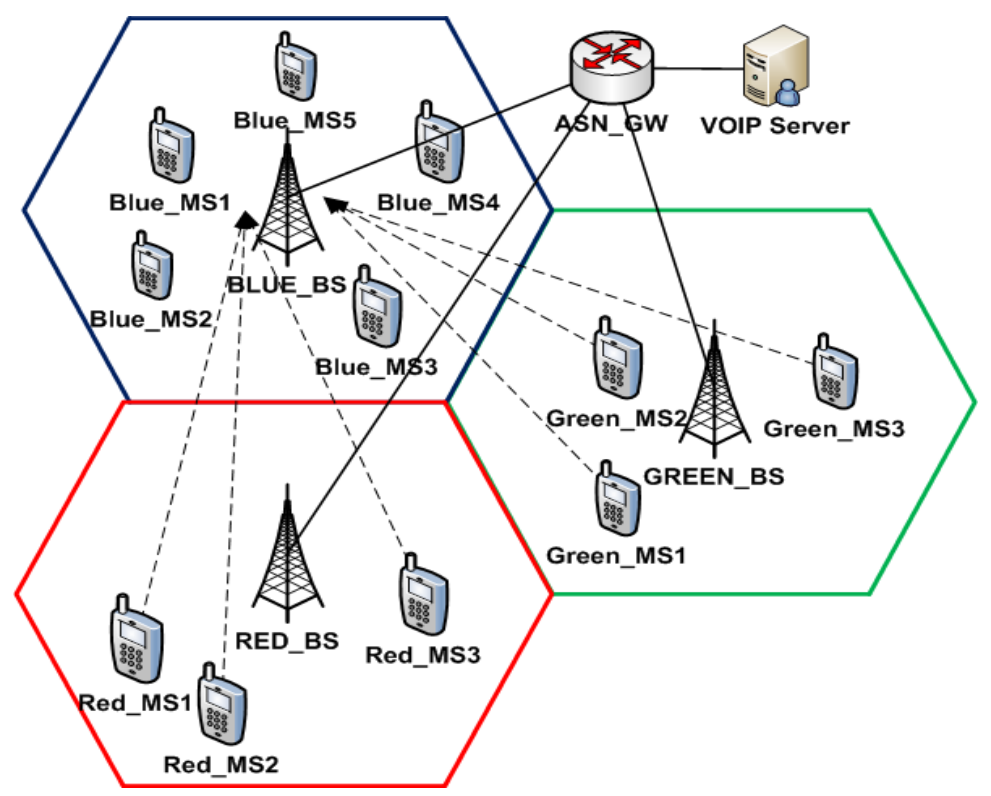

Fig. 2. WiMAX Network Topology for Handover simulation

Blue MS nodes are static, Red and green MS nodes have trajectories that start movement on trajectories to reach Blue BS. This simulation executed without any load balancing technique and the SBS ID observed that corresponds to each MS during the simulation. Blue_MS nodes are attached to the BS with ID 7 (Blue_BS). Green MS nodes are initially attached to the BS with ID 3 (Green_BS). At approximately 20 minutes, as they moved towards the blue cell, they switch to BS_ID 7 (Blue_BS). Red MS nodes are initially attached to the BS with ID 0 (Red_BS). At approximately 18 minutes they moved towards the blue cell, they switched to BS ID 7 (Blue_BS).

The free uplink capacity has been observed on Blue_BS during the handover of the green and red nodes in Fig. 3. As the MS nodes attached to Blue_BS, the estimated free uplink resources in the cell are reduced. Each MS requires approximately 70 Ksps 


\section{A. S. Abdel-Rahman, N. Semary, H. S. Abdelkader}

(kilo symbols per second) for its UGS uplink connection the uplink resources are exhausted around 15 minutes. Uplink resources start freeing up as the MS nodes leave Red_BS and Green_BS to join Blue_BS. Uplink resources are freed once the retain timer expires (after handover is completed).

The application throughput observed on each group of nodes. In Blue_MS nodes, all the MSs have the same throughput approximately $100 \mathrm{Kbps}$ as shown in Fig. 4 . In the period from 14 to 18 minutes, the throughput reduced slightly because in this period the Red_MS nodes and Green_MS nodes tried to associate with BLUE_BS. And throughput decreased during initial ranging activity for Red_MS nodes in Fig. 5. In Fig. 6 throughput decreased during initial ranging activity for Green_MS nodes.

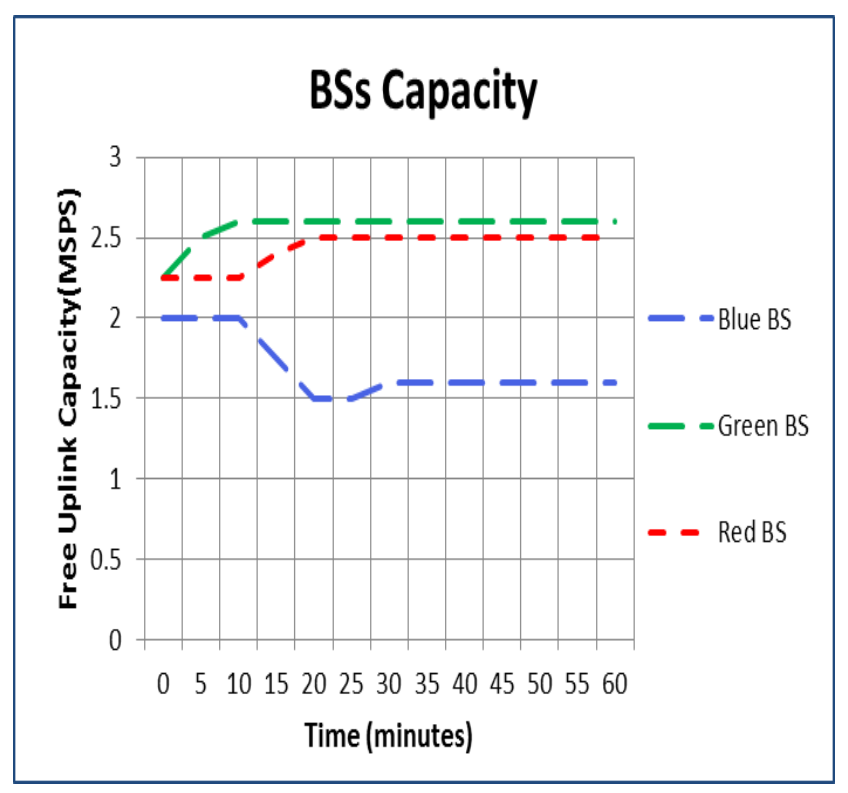

Fig. 3. Free uplink capacity on all BSs in simulated network

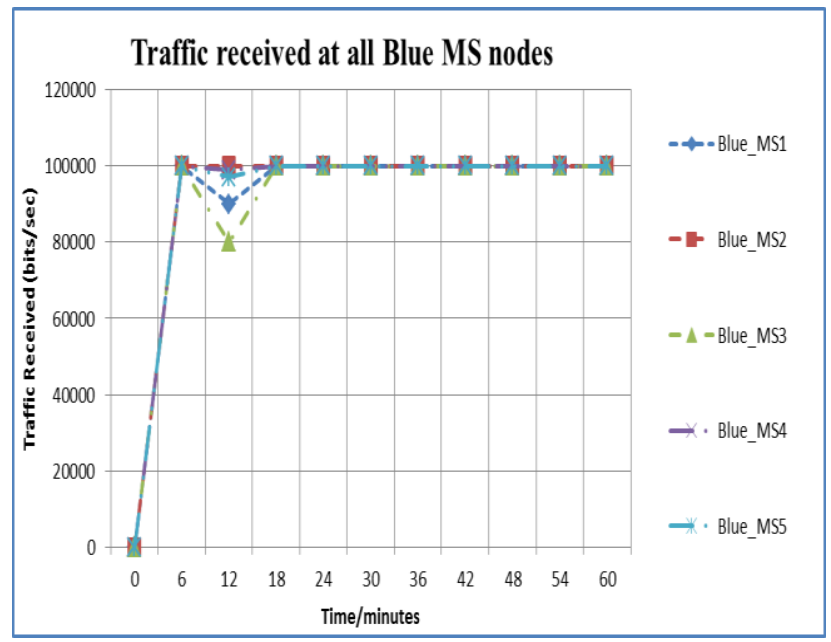

Fig. 4. Traffic received at Blue_MS nodes 


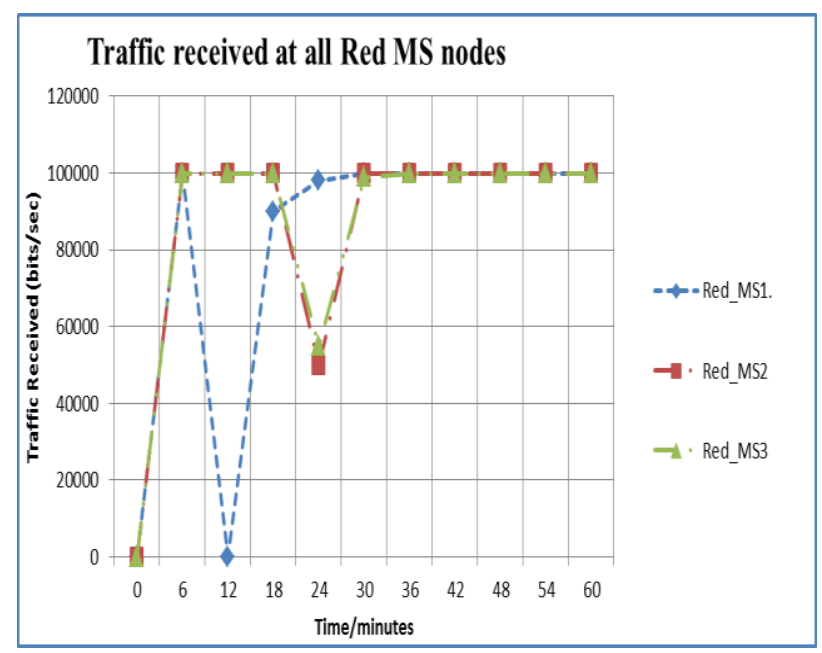

Fig. 5. Traffic received at Red_MS nodes

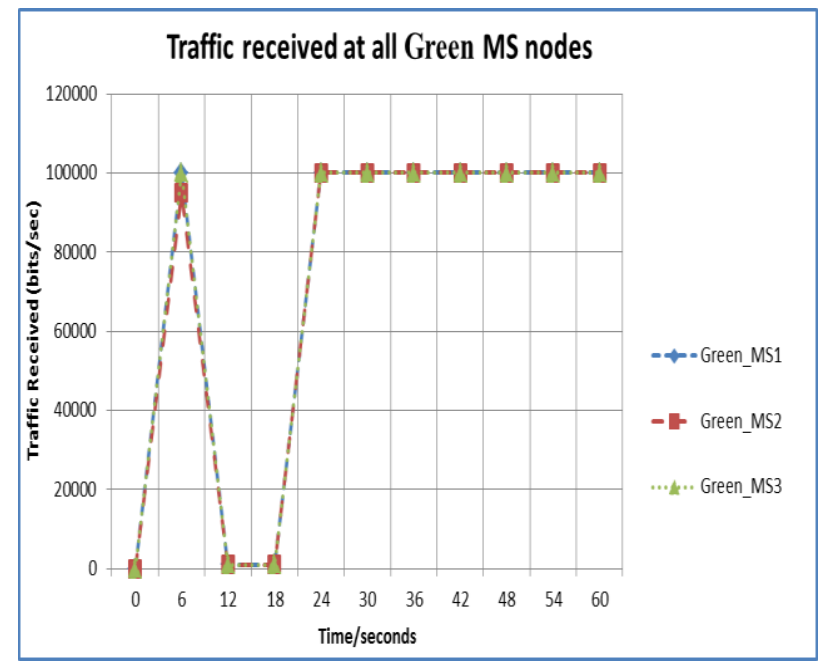

Fig. 6. Traffic received at Green_MS nodes

In load balancing technique using SCP technique, BLUE_BS decided to distribute load with neighbor BSs. BLUE_BS forced MS in overlapping area to attach to Target BS. Blue _MS 4 and Blue_ MS 5 attached with GREEN_BS with ID 3 because it is closer to them. Blue_MS 2 and Blue_MS 3 attached to RED_BS with ID 0 because it is closer to them. Blue_MS 4 and Blue_MS 5 transferred to BS_ID 3 (GREEN_BS), Blue_MS 2 and Blue_MS 2 transferred to BS_ID 0 (RED_BS). Free uplink capacity after forced handover increased for BLUE_BS and the decreased for RED_BS and GREEN_BS this means that load balancing occurs with BLUE_BS with RED_BS and GREEN_BS as shown in Fig. 7. In Fig. 8 (a,b) and c showed the traffic received at all MS nodes in three cells using SCP technique. Traffic received for Blue_MS nodes decreased in the period of handover from BLUE_BS to RED_BS and GREEN_BS.

In the second scenario, Network topology is the same as in the first scenario. Forced handover examined using WQLB procedure [ HYPERLINK $\backslash 1$ "amiri2012wqlb" 10 ]. Delay and throughput observed for uplink connection in all MS nodes for all BSs. In Fig. 9 we observed that MS nodes in BLUE_BS have low throughput. From Fig. 10, we can observe that Blue_MS 2, Blue_MS 3, Blue_MS 4 and Blue_MS 5 delay approximately is 0.12 second. This means that BLUE_BS choose MS nodes that reside in overlapping area to execute forced handover with neighbor BS to distribute load with neighbor BSs to guarantee all service classes of QoS in Mobile WiMAX Network. We observe the uplink free capacity of the three BSs after forced handover using WQLB 
A. S. Abdel-Rahman, N. Semary, H. S. Abdelkader

technique, we get that free uplink capacity in BLUE_BS increased and free uplink capacity in RED_BS and GREEN_BS decreased.

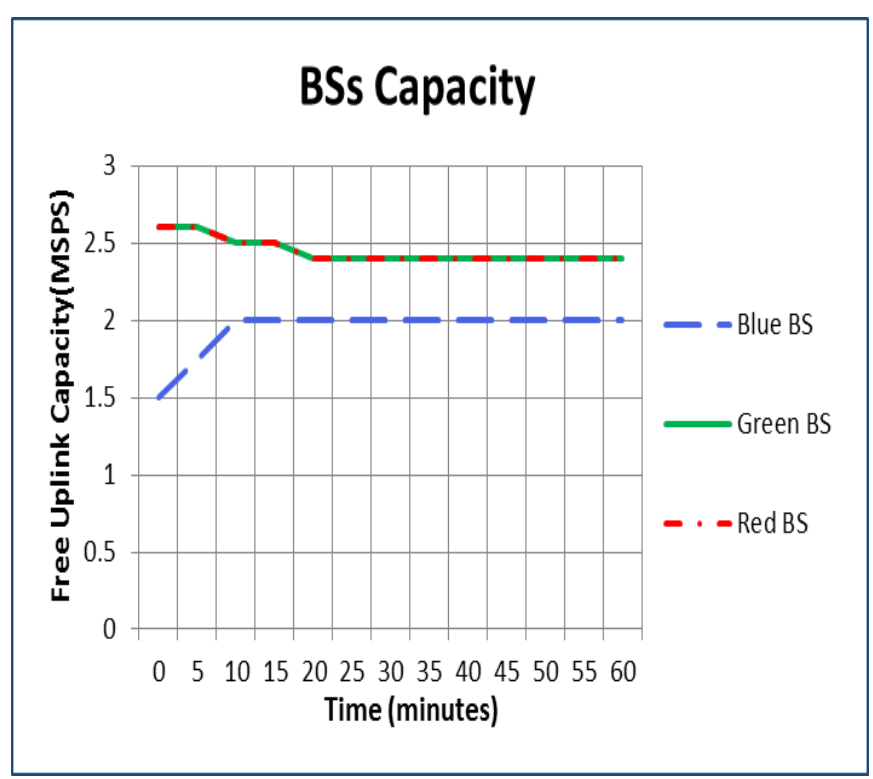

Fig. 7. Free uplink capacity for all BSs

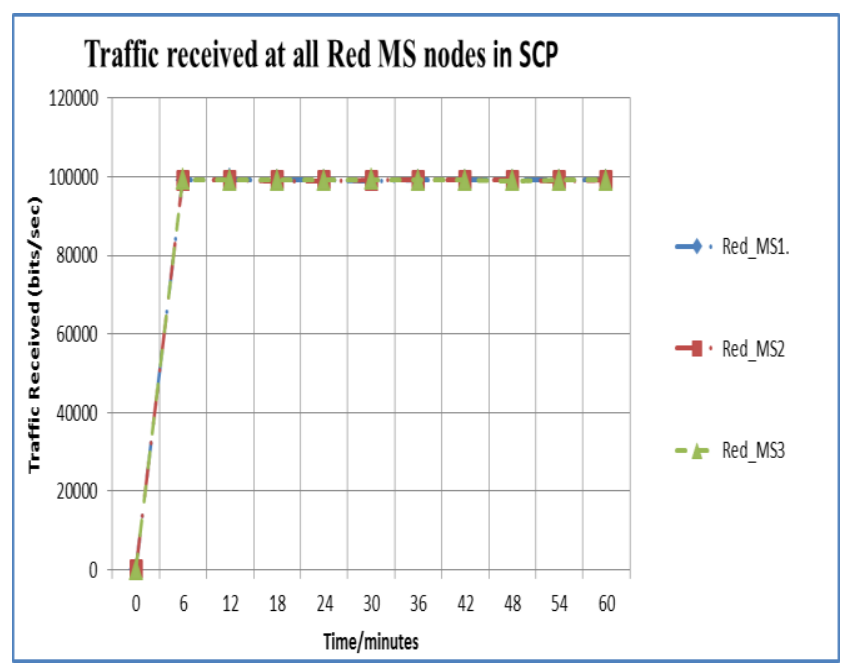

(a) 


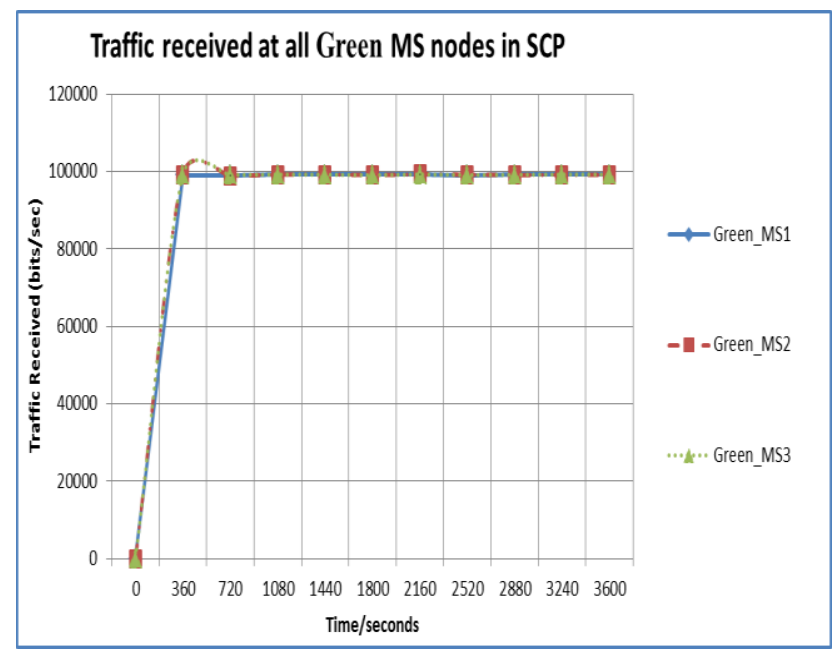

(b)

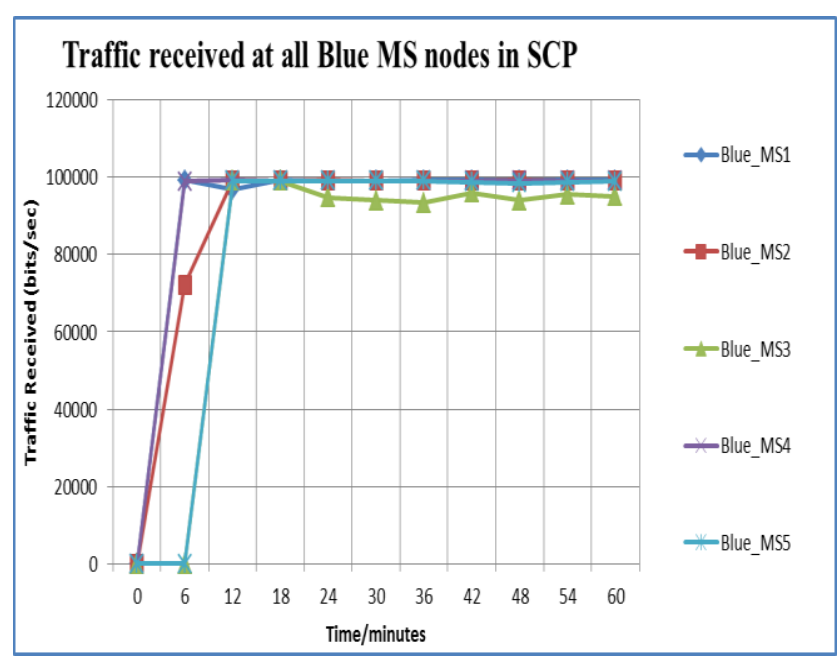

(c)

Fig. 8. Traffic received at all MS nodes in SCP.

As shown in Fig. 11, the free uplink capacity of RED_BS, GREEN_BS and BLUE_BS becomes approximately equal. Throughput of all Red and Green MS nodes is shown in Fig. 12 (a,b). Throughput reduced for Red_MS nodes and Green_MS nodes reduced due to forced handover delay. In handover operation MS nodes break connection from serving BS and make connection with target BS. As shown in Fig. 11, the three BSs free uplink capacity becomes approximately equal. This means that WQLB can load balance in the whole network efficiently. WQLB can manage BS resources efficiently. Throughput of all Red and Green MS nodes is shown in Fig. 12 (a, b). Throughput reduced for Red_MS nodes and Green_MS nodes reduced due to forced handover delay. 
A. S. Abdel-Rahman, N. Semary, H. S. Abdelkader

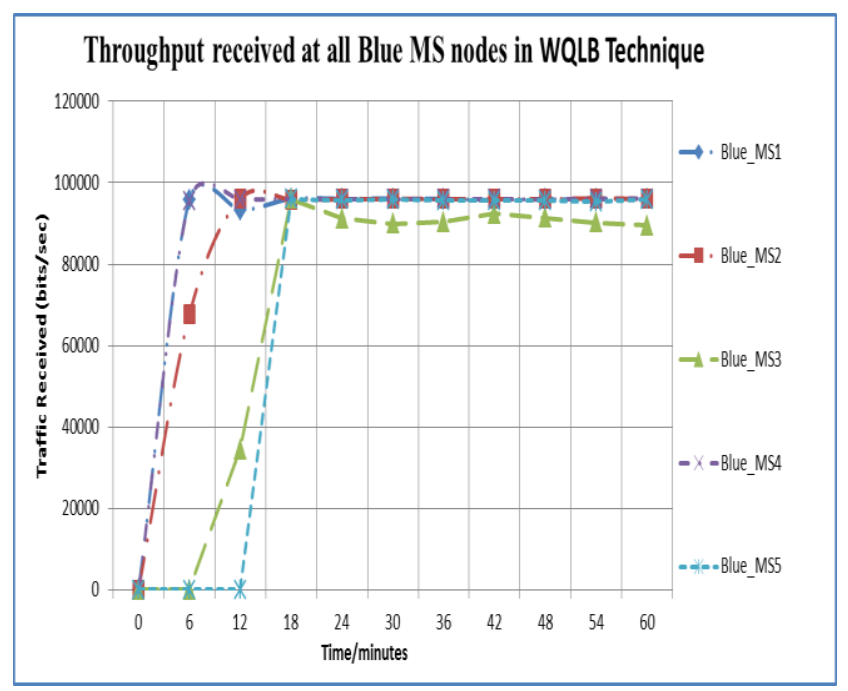

Fig. 9. Throughput for Blue_MS nodes in WQLB technique

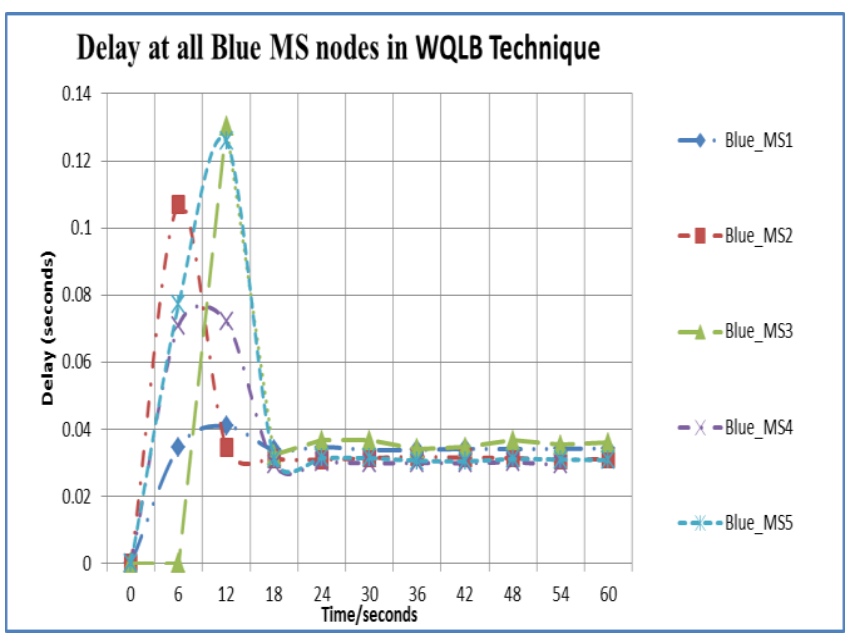

Fig. 10. Delay for BLUE_MS nodes in WQLB technique

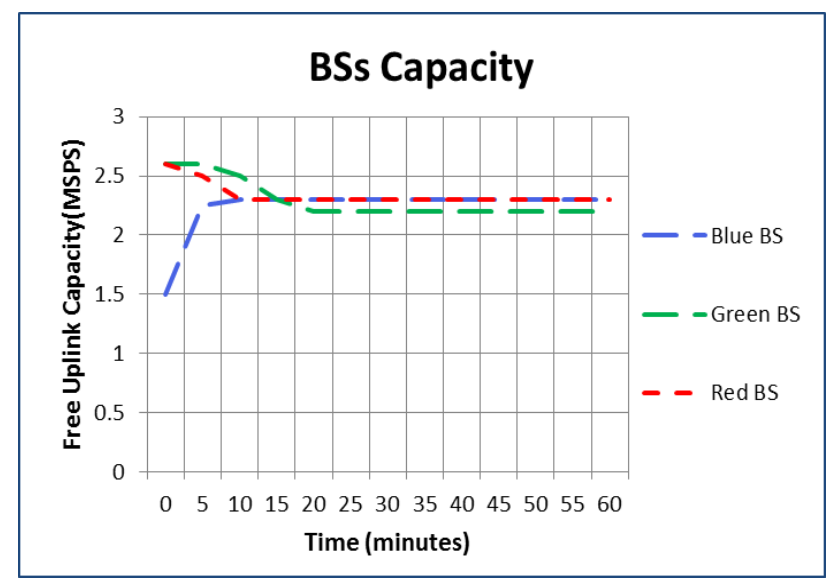

Fig. 11. Free Uplink Capacity for all BSs WQLB technique 
IJCI Vol. 4 - No. 1, July 2015

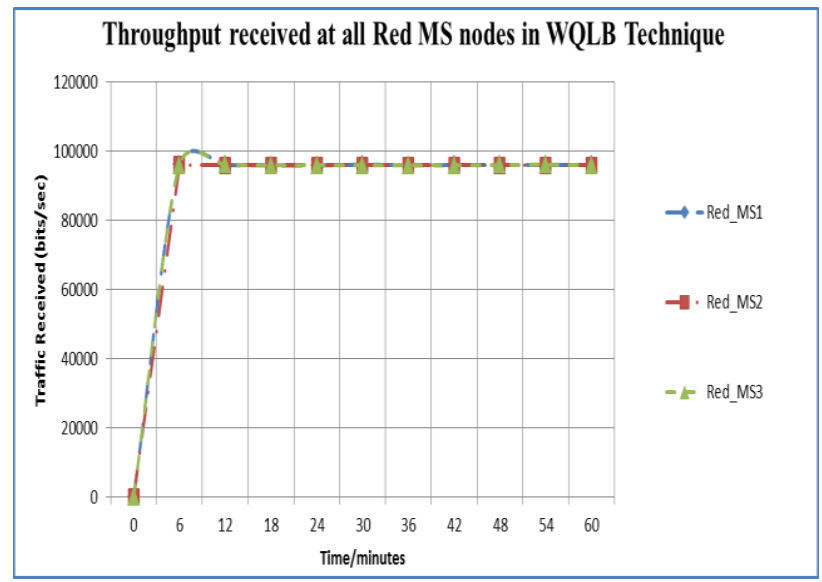

(a)

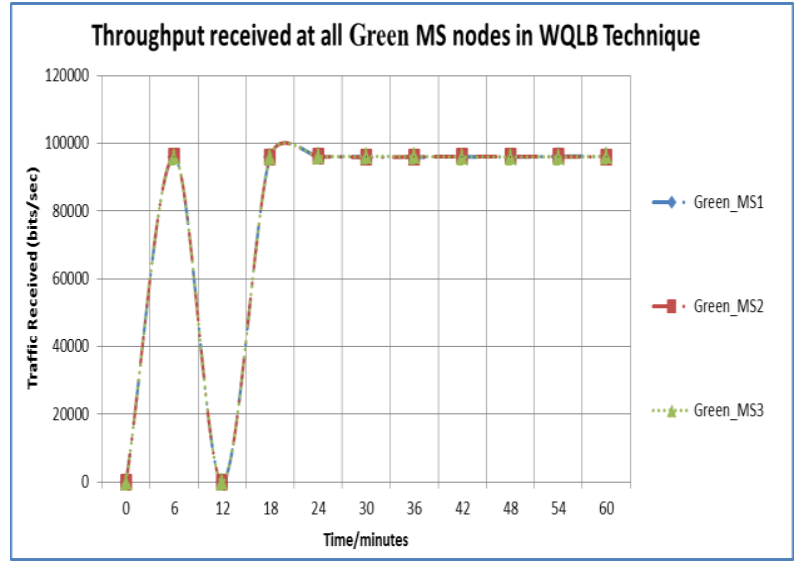

(b)

Fig. 12. Throughput for all MS nodes in WQLB technique

\section{IV.CONCLUSION}

In this paper we have examined two Base Station controlled handover based load balancing in Mobile WiMAX system. Based on the two schemes examined in this paper, the load balancing evaluated. The simulation results showed that the two schemes can balance load and enhance system wide resource utilization. In WQLB scheme the free uplink capacity at all BSs are approximately equal that means it can distribute load in the whole network efficiently, but throughput in SCP scheme is better than WQLB. In SCP the pre-handover control messages make handover process consume small time and TBS can distinguish whether handover is a rescue or directed, this help to execute handover with high priority. In WQLB, directed handover take more time than SCP technique, so the throughput in handover time approximately zero. SCP technique performs better than WQLB technique for real time applications; SCP pre-handover control messages reduce handover time, reduce packet loss and guarantee handover acceptance from neighbor BS. Pre-handover messages don't exist in WQLB technique handover as in SCP technique; delay and packet loss in WQLB is larger than SCP. WQLB technique performs better than SCP technique in balancing load at the whole network and BS resource management. 


\section{REFERENCES}

[1] T. Casey, N. Veselinovic, and R. Jantti, "Base Station Controlled Load Balancing with Handovers in Mobile WiMAX," in Personal, Indoor and Mobile Radio Communications, 2008. PIMRC 2008. IEEE 19th Intern ational Symposium on, Sept 2008, pp. 1-5.

[2] "IEEE Standard for Local and Metropolitan Area Networks Part 16: Air Interface for Fixed Broadband Wireless Access Systems," IEEE Std 802.16-2004 (Revision of IEEE Std 802.16-2001), pp. 0_1-857, 2004.

[3] "IEEE Standard for Local and Metropolitan Area Networks Part 16: Air Interface for Fixed and Mobile Broadband Wireless Access Systems Amendment 2: Physical and Medium Access Control Layers for Combined Fixed and Mobile Operation in Licensed Bands and Corrigendum 1," IEEE Std 802.16e-2005 and IEEE Std 802.16-2004/Cor 1-2005 (Amendment and Corrigendum to IEEE Std 802.16-2004), pp. 0_1-822, 2006.

[4] S.K. Ray, K. Pawlikowski, and H. Sirisena, "Handover in Mobile WiMAX Networks: The State of Art and Research Issues," Communications Surveys Tutorials, IEEE, vol. 12, no. 3, pp. 376-399, Third 2010.

[5] S. Tang, P. Muller, and H.Sharif, WiMAX security and quality of service: an end-to-end perspective.: John Wiley I\& Sons, 2011.

[6] A. et al Agis, "Global Interoperable Broadband Wireless Networks:Extending WiMAX Technology to Mobility".: Intel Technology Journal, 2004.

[7] Q. Hu, D. Paranchych, M. Fong, and G. Wu, "On the evolution of handoff management and network architecture in WiMAX," in Mobile WiMAX Symposium, 2007. IEEE, 2007, pp. 144-149, 2007.

[8] A. Khatkar, Y. Singh, and V. Nandal, "A QoS Oriented Analysis of ertPS, rtPS and nrtPS flows in WiMAX," International Journal of Advanced Research in Computer Science and Software Engineering, vol. 3, no. 7, pp. 85-90, July 2013.

[9] C. Askarian and H. Beigy, "A Survey for Load Balancing in Mobile WiMAX Networks," Advanced Computing: An International Journal, vol. 3, no. 2, pp. 119-137, 2012.

[10] C. Askarian and S. M. Aghdam, "WQLB: WiMAX QoS Aware Load Balancing Protocol," in Computer and Information Technology (CIT), 2012 IEEE 12th International Conference on, 2012, pp. 615-622, 2012.

[11] S. Moiseev et al., "WLC17-4: Load-Balancing QoS-Guaranteed Handover in the IEEE 802.16e OFDMA Network," in Global Telecommunications Conference, 2006. GLOBECOM '06. IEEE, Nov 2006, pp. 1-5, 2006.

[12] S. H. Lee and Y. Han, "A Novel Inter-FA Handover Scheme for Load Balancing in IEEE 802.16e System," in Vehicular Technology Conference, 2007. VTC2007-Spring. IEEE 65th, April 2007, pp. 763-767, 2007.

[13] K. P. Moon, J. Park, J. Kim, H. Choo, and M. Chung, "Dual Handover Procedures for FA Load Balancing in WiBro Systems," in Convergence and Hybrid Information Technology, 2008. ICCIT '08. Third International Conference on, vol. 2, Nov 2008, pp. 54-59, 2008.

[14] J.J. Bazzo, AM. Cavalcante, M.J. de Sousa, L. Kuru, and J. Moilanen, "Load Balance for Multi-Layer Reuse Scenarios on Mobile WiMAX System," in Vehicular Technology Conference Fall (VTC 2010-Fall), 2010 IEEE 72nd, Sept 2010, pp. 1-5, 2010.

[15] S. Nazari and H. Beigy, "A new distributed uplink packet scheduling algorithm in WiMAX newtorks," in Future Computer and Communication (ICFCC), 2010 2nd International Conference on, vol. 2, May 2010, pp. V2-232-V2-236, 2010.

[16] Y. Ahmet , M. Ivanovich, and A. Ye, "A survey of MAC based QoS implementations for WiMAX networks," Computer Networks, vol. 53, no. 14, pp. 2517-2536, 2009.

[17] Frank Ohrtman, WiMAX Handbook: Building 802.16 Networks.: McGraw-Hill Communications, 2005.

[18] S. Siraj, A. Gupta, and R. Badgujar, "Network simulation tools survey," International Journal of Advanced Research in Computer and Communication Engineering, vol. 1, no. 4, pp. 199-206, 2012. 